\title{
De Europa a África pasando por Indochina
}

Guías de conversación plurilingües para viajeros del siglo xIX y algunas notas sobre los manuales de lenguas de regiones colonizadas

\section{Luis Pablo Núñez}

\section{OpenEdition}

Journals

Edición electrónica

URL: https://journals.openedition.org/cher/2989

DOI: $10.4000 /$ cher.2989

ISSN: 2803-5992

Editor

Presses universitaires de Strasbourg

\section{Edición impresa}

Fecha de publicación: 27 junio 2017

Paginación: 153-169

ISBN: 978-2-86820-961-0

ISSN: 1968-035X

\section{Referencia electrónica}

Luis Pablo Núñez, «De Europa a África pasando por Indochina», reCHERches [En línea], 18 | 2017,

Publicado el 01 diciembre 2021, consultado el 22 diciembre 2021. URL: http://

journals.openedition.org/cher/2989; DOI: https://doi.org/10.4000/cher.2989

\section{(C) $(100$}

Ce(tte) œuvre est mise à disposition selon les termes de la Licence Creative Commons Attribution Pas d'Utilisation Commerciale - Partage dans les Mêmes Conditions 4.0 International. 


\title{
De Europa a África pasando por Indochina: guías de conversación plurilingües para viajeros del siglo XIX y algunas notas sobre los manuales de lenguas de regiones colonizadas
}

\author{
LUIS PABLo NúNEEZ*
}

\section{Propósito de este trabajo}

Este trabajo tiene como objetivo la reflexión sobre los modelos gramaticales empleados en obras del siglo xIx a la hora de describir una lengua europea, $y$ los procesos de interferencia, calco, hibridismo o semejanza estructural usados al describir la gramática de otra lengua tipológicamente distinta con la que se pone en contraste.

Para ello describiremos contraponiéndolas entre sí algunas obras decimonónicas: por un lado, los manuales plurilingües para viajeros del siglo XIX con lenguas europeas que se basaron en los diálogos y las nomenclaturas como método de aprendizaje, representados a través de la guía de conversación en siete lenguas de Melchor Nuñez de Taboada, publicada en París en 1833 (Guide de la conversation, ou, Vade-mecum du voyageur, en sept langues, anglais, allemand, français, italien, espagnol, russe, barbaresque ou moresque); por otro lado, varios títulos contemporáneos bilingües en los que se describe el léxico o la gramática de lenguas no europeas, más concretamente africanas y del sudeste asiático, en el momento de las colonizaciones de aquellos territorios por las naciones europeas.

Las preguntas que queremos plantear son: ¿cómo se enfrentaron los europeos a otras lenguas culturalmente alejadas que no contaban con manifestaciones escritas y nunca antes habían sido codificadas? ¿Hasta qué punto se traspuso el método usado en la descripción de las lenguas occidentales a aquellas lenguas de

* Profesor ayudante doctor en el Departamento de lengua española de la Universidad de Granada. 
las regiones colonizadas? ¿Se adaptaron las descripciones de aquellas lenguas a los apartados habituales en la descripción de lenguas europeas?

La contraposición que realizamos aquí no tiene como propósito hacer un análisis exhaustivo de estas obras: somos conscientes de que cada una de ellas merecería un estudio independiente y detallado; del mismo modo, las obras sobre lenguas no europeas habrían de ser puestas en correlación con otras similares lingüísticamente o de otros periodos históricos. Nuestro propósito es partir de la cercanía cronológica en que se publicaron unas y otras y mostrar, desde una perspectiva comparativa, semejanzas o diferencias.

Es importante señalar que, frente a las obras del siglo XVI -diccionarios y gramáticas amerindios-, ampliamente estudiados por la Historiografía lingüística y la Lingüística misionera, las obras tocantes a los periodos posteriores -siglos XVIII y XIX, momentos de las exploraciones oceánicas del Pacífico y luego de las colonizaciones-, a pesar de su mayor proximidad cronológica a nuestros días, no han sido apenas analizadas, al menos en la historiografía lingüística española. Este artículo y otros que esperamos presentar más adelante quiere defender esta línea de investigación y paliar esta carencia en nuestros estudios.

\section{Manuales de lengua del siglo xIx: las guías de conversación}

El siglo XIX supone en Europa la consolidación de la imprenta a escala industrial y la aparición de nuevas figuras en el ámbito editorial, los editores y las editoriales en nuestro sentido moderno. El incremento de la producción libraria debido a las nuevas técnicas mecánicas será patente a medida que avance el siglo no solo en el aumento del número de impresos populares, de las gacetas y de los papeles periódicos, de las novelas de folletín, sino también en la elaboración de los diccionarios y en los métodos para el aprendizaje de lenguas.

La aparición de una clase burguesa, la mejora de las comunicaciones y del transporte -por tierra o por mar, muy especialmente el ferrocarril y el barco de vapor ${ }^{1}-\mathrm{y}$ el aumento de los contactos comerciales fomentó también unos tipos de obras que ya existían de alguna manera en siglos anteriores, pero no de forma tan consolidada: las guías de viaje, por un lado, y, a finales de siglo, los manuales de correspondencia comercial, por otro (por ejemplo, el Manual de la correspondencia comercial en dos lenguas: española y alemana de Hermann Robolsky y Santo Domingo publicado en Leipzig por G. A. Gloeckner, 1887, o el Manual of Mercantile Correspondence in Two Languages - English and German de Ludwig Simon et al., Gloeckner, 1883).

1 El transporte de pasajeros en ferrocarril se realizaba ya en 1830 en Mánchester y Liverpool, en Bélgica el ferrocarril se inauguró en 1835, y Francia tuvo su primera línea en 1836. Pocos años después, en 1850, Alemania ya contaba con $4500 \mathrm{~km}$ de vías férreas y Francia con 3000. En la misma década de 1830, un barco de vapor realizó por primera vez un viaje por el Atlántico Norte. 
Paralelamente a lo que veremos en la segunda parte de este artículo, las guías de viaje publicadas a comienzos del siglo xix son una muestra muy representativa de la nueva escala económica y colonial del momento. Las Murray Handbooks for travellers del editor británico John Murray III (1808-1892), señaladas como las primeras guías turísticas (Lyons 2011: 139-140), llegaron a ser tan conocidas que ya las reseñas de época las consideraban como propias de un imperio:

Murray's Guidebooks now cover nearly the whole of the Continent and constitute one of the great powers of Europe. Since Napoleon no man's empire has been so wide. From St. Petersburg to Seville, from Ostend to Constantinople, there is not an inn-keeper who does not turn pale at the name of Murray (Lister et al. 1993: ix).

Las publicadas por el editor Karl Baedeker (1801-1859) en Coblenza y Leipzig fueron igualmente famosas, con una variedad de títulos que abarcaban prácticamente todos los países y regiones de Europa y una gran cantidad de ediciones. Su primer título se publicó en 1835 y su éxito fue inmediato: por ejemplo, en 1881 se habían publicado diez ediciones del Manuel du voyageur. Belgique et Hollande, aparecido pocos años antes.

Otras colecciones de guías de viaje eran las publicadas en Würzburg y Viena, la Woerl's Reisehandbücher, cuyo catálogo incluía en 1885 títulos sobre diversas regiones de Europa o Constantinopla (Estambul), Norte de África, México, Palestina, Sudamérica, California y Egipto.

Todas tomaban el mismo formato: libros de pequeño tamaño, fáciles de transportar, con encuadernación rígida roja con letras doradas y mapas desplegables. Su tipología impresa sería tan característica del género que las guías de décadas posteriores seguirían adoptando la encuadernación roja, como los manuales de conversación de los Hermanos Garnier de 1900.

Estas guías decimonónicas, ampliamente documentadas, no solo indicaban las informaciones históricas, culturales e itinerarios que hoy encontramos en cualquier guía de nuestros días, sino también el plan de viaje, los gastos en cada una de las etapas y el tiempo necesario para recorrerlas $-y$, en ocasiones, incluso, el tipo de ropa que vestir y el número de prendas que se recomendaba llevar-.

Las guías también incluían notas lingüísticas de los lugares en un apartado específico sobre «Lengua». Se trataba de breves nociones gramaticales y algunas frases prácticas. Para las mayores necesidades comunicativas de los viajeros se publicaron guías específicas de conversación en varias lenguas, como el Traveller's manual of conversation in four languages, English, French, German, Italian. With vocabulary, short questions, etc. (Leipzig, K. Baedeker, 1875 la 22. ${ }^{\text {a }}$ edición)².

2 En una de las hojas de guarda de las guías Baedeker, se anuncia el Manuel de Conversation pour le touriste en quatre langues (français, allemand, anglais, italien), avec un vocabulaire, un choix de question diverses, etc. La 17. ${ }^{\text {a }}$ ed., en dozavo, se publicó en 1864; la 18. a en 1866. 


\section{Langue.}

La langue hollandaise est une variété de la branche appelée bas-allemand; elle ne se distingue que dans quelques détails insigniflants de la langue flamande (v. p. 2). Tandis que l'idiome des Flandres et du Brabant est resté stationnaire sous les gouvernements étrangers qui ont possédé ces pays, le dialecte hollandais s'est constitué en langue littéraire et grammaticalement réglée. Les influences romanes s'y font sentir et des mots comme kantoor (comptoir), kwartier (quartier), kapitein (capitaine), rekwest (requête) et beaucoup d'autres emprunts faits a la langue française, se rencontrent en foule dans le dictionnairo hollandais. Les Hollandais peuvent se glorifler d'une littérature riche en productions de tout genre.

La langue française suffit généralement au voyageur ordinaire, surtout dans les endroits fréquentés d'habitude par les étrangers; mais il est bon néanmoins, comme partout, d'avoir au moins quelque notion de la langue, de connaitre les mots indispensables pour les relations forcées avec les gens du peuple, mots qu'on trouve, par exemple, dans un Guide de la conversation français-hollandais.

Cependant, comme les livres sur la langue hollandaise à l'usage des Français sont assez rares, le voyageur ne sera sans doute pas fâché de trouver ici quelques données sur la prononciation de cette langue, un certain nombre de mots indispensables et de petites phrases blémentaires. La connaissance de l'allemand aide naturellement à comprendre le hollandais.

Prononolation, en tant qu'elle présente des différences essentielles d'avec celle du français. U se prononce à peu près ou et quelquefois $\mathrm{u} ; v, \mathrm{f} ; a a, e e, o o, u u, \hat{\mathrm{a}}, \hat{\boldsymbol{\theta}}, \hat{\mathbf{b}}, \hat{\mathrm{u}} ; e i, i j$, ou $y$, eí ; par ex., dans mijnheer, monsieur (ménère); ie, $\hat{\text {; }}$ oe, ou; au, ou, ouw, à peu près comme aou, par ex. dans mevrouw, madame (méfraou); jungjuffrouw et juffrouw, mademoiselle (ajuffrouw $D$ se dit aux buffets ot dans les magasins, même aux dames mari6es).

Article, avec lequel on décline les substantifs et les adjectifs (en ou $s$ au pluriel). Singulier. Nominatif: de, le, la; het, le (neutre). Génitif: des, der, des ou van den, van de, van het, du, de la, du. Datif: den, der, den ou aan den, aan de, aan het, au, à la, au. Accusatif : den, de, het, le, la, le. Pluriel: de, les; der ou van de, des; den ou aan de, aux; de, les, pour les trois genres.

Baedeker, Manuel du voyageur. Belgique et Hollande... pp. 204 y 205. 


\section{Phrages et termes useels.}

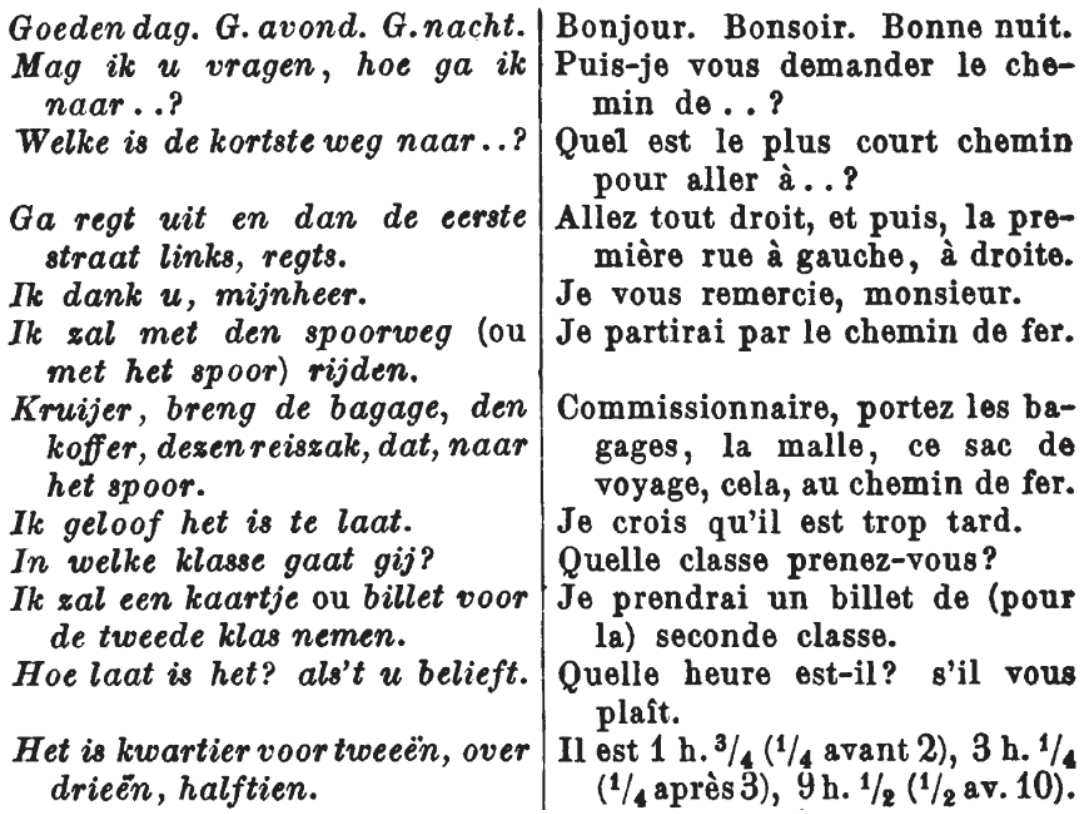

Baedeker, Manuel du voyageur. Belgique et Hollande...

Frases útiles neerlandés-francés, pp. 206-208.

Las Baedeker o las Murray fueron las guías más conocidas, pero anteriormente existieron obras similares bajo otros modelos. Las guías de viaje se pueden considerar la evolución decimonónica lógica, en un contexto histórico y social nuevo, de los manuales de conversación que se imprimían durante los siglos XVI, XVII y XVIII para comerciantes y viajeros.

$\mathrm{Y}$ en el XIx estas necesidades comerciales continuaron. Por ejemplo, los intereses comerciales de Portugal con Francia e Inglaterra fueron la causa de la publicación de la Collecção de phrases e dialogos familiares uteis aos portuguezes, francezes e inglezes; ou, Exercicios para a conversação portugueza, franceza e ingleza (Lisboa, Impressão Regia, 1829) de Emilio Achilles Monteverde.

$\mathrm{O}$, en esa línea de guías de conversación mencionada, la que motivó la aparición de esta otra en la que nos centraremos ahora, la de Melchor Manuel Nuñez de Taboada, titulada Guide de la conversation, ou, Vade-mecum du voyageur, en sept langues, anglais, allemand, français, italien, espagnol, russe, barbaresque ou moresque; contenant des conversations familières sur divers besoins et circonstances où il peut se trouver. Ouvrage mis en ordre, revu et corrigé sous la direction de M. Nuñez de Taboada... Suivi d'un vocabulaire français 
arabigo-barbaresque, et d'un dialogue dans ces deux langues sur des choses de première nécessitè par M. Nuñez de Taboada (Paris, Thiériot, 1833, 180 p.).

Núñez de Taboada, que realizó un Diccionario de la lengua castellana (París, 1825), pero era especialmente conocido por un diccionario frances-español y español-frances que tuvo mucho éxito ${ }^{3}$, fue el encargado de coordinar, revisar y corregir esa guía de conversación de 1833.

Esta es la edición que hemos manejado. No obstante, un extracto de 36 páginas con los primeros diez capítulos se incluyó también parcialmente, como anexo o como reclamo, a otros títulos publicadas por el mismo editor Thiériot: así, se incluyó en el Nouveau dictionnaire de poche français-italien/italienfrançais de Briccolani (1833, 12 éd. 1840) y en el Nouveau dictionnaire de poche français-allemand et allemand-français de Zay (1840).

La guía de conversación se compone en primer lugar de varios apartados breves con forma de nomenclatura (fórmulas de cortesía / meses del año / días de la semana / números) y sigue con 26 apartados de conversación relativos a viajes por mar o por tierra, frases para pedir de comer en una posada, para comprar o lavar ropa, para contratar un criado o portador de equipaje y para hablar con otras personas a las que se encuentra en el camino.

Siguen a continuación once apartados sobre modelos de cartas de negocio (p. 143-154: recibos, carta a un banquero solicitando dinero, billete para excusarse de no poder asistir a un convite, etc.) donde se reflejan las convenciones sociales. Las fórmulas habituales se dan por sabidas («Tengo el honor de ser, etc.») y no se desarrollan en toda su extensión.

Finalmente se incluye un vocabulario francés-árabe y un diálogo francésárabe. Este «diccionaire français-barbaresque ou moresque», que abarca 20 páginas, recoge el léxico más usual en francés y su equivalencia en la otra lengua. No es propiamente un diccionario, como señala en p. 156-157:

Ce Vocabulaire, qui n'est pas un Dictionnaire proprement dit, renferme cependant les mots les plus usuels et les plus nécessaires à connaître, lorsqu'on voyage dans un pays quelconque dont on ignore la langue. Les correspondances arabicobarbaresques ou moresques sont figurées en caractères romains aussi exactement qu'il est possible de figurer la vraie prononciation d'une langue. [...] on peut être assuré de se faire bien comprendre des barbaresques ou moresques, surtout des Algériens.

3 La primera edición se publicó en París, Brunot-Labbe Libraire, 1812, y de él se hicieron numerosas reimpresiones: la 14. ${ }^{a}$, por ejemplo, en 1859. Tanto éxito tuvo este diccionario bilingüe que se publicó de forma pirata bajo su nombre una versión abreviada titulada Nouveau dictionnaire de poche français-espagnol. El «Avis des éditeurs» en las ediciones de mediados del siglo XIX advierte de la falsificación: «C'est un devoir pour nous de prévenir qu'on a publié à Paris un petit Dictionnaire de poche espagnol-français et français-espagnol sous le nom de M. Nuñez de Taboada, bien qu'il n'en soit pas l'auteur. Les tribunaux ont condamné les éditeurs de ce Dictionnaire, et consacré nos droits par un jugement authentique» (Avis, ed. de Paris, En casa de P.-J. Rey, Librero, 1845). 
Así, algunas entradas son los números de acuerdo con el orden alfabético en donde les corresponde:

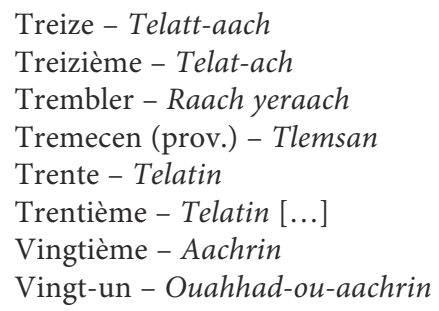

En cuanto al diálogo francés arabico-barbaresque, no es más que una página y media de frases para establecer el contacto y preguntar si tiene comida y se la vende, con la transcripción en caracteres latinos en otra columna de las equivalencias en lengua árabe:

Entendez-vous l'arabe?

Un peu

J'ai faim

Donnez-moi à manger

Donnez-moi de l'eau

Donnez-moi du pain

Avez-vous de la viande?

Avez-vous des légumes?

Vendez-m'en, je vous prie; je paierai comptant.

De lo descrito podemos resumir lo siguiente: la estructura es parcialmente dialógica, con predominio de frases y expresiones sueltas, no diálogos como los que encontrábamos en siglos anteriores. Las frases se agrupan por ámbitos temáticos y se atiende a cuestiones prácticas (salarios, caminos).

Aunque la guía incluye varias lenguas tipológicamente diversas entre sí, como el ruso, en ningún momento se describe siquiera unas nociones mínimas de transliteración, no hay descripción gramatical ni explicación del léxico: solo hay frases de equivalencia. Las situaciones comunicativas eran lo fundamental de estas obras, como también veremos que ocurre en los manuales sobre lenguas no europeas.

\section{La colonización y las lenguas no europeas}

Al mismo tiempo que se imprimían estas guías de viaje y guías de conversación para lenguas de Europa, las naciones inglesa y francesa exploraban en el siglo XIX los mares y los continentes continuando las expediciones que se habían llevado a cabo a finales del siglo xvirI.

La llegada de los europeos a los confines de la Tierra y la instauración de emporios comerciales que luego dieron pie a la colonización supuso el encuentro con otras muchas culturas sobre las que se ejerció la dominación. No obstante, ya fuera por imponer esa dominación, por la más rápida explotación de los 
recursos de aquellos territorios, por evangelizar a sus habitantes o por curiosidad científica y etnográfica, el conocimiento de la lengua de sus habitantes fue también valorada y tenida en cuenta.

La difusión de la cultura y la imposición de la civilización europea -«on était animé à Paris et à Londres des mêmes vues et de la même sollicitude pour la connaissance des langues de l'Afrique et l'amélioration du sort des indigènes" (Dard 1825: xiv)- escondía al mismo tiempo una forma de competencia colonial entre Francia e Inglaterra, como se ve en este fragmento:

Il est remarquable que c'est dans le même temps qu'on songeait, en France et en Angleterre, à se familiariser avec les dialectes de l'Afrique, dans la vue de former de jeunes noirs à la connaissance des langues et des sciences européennes, et de les rendre propres à observer leur pays, à rediger leurs observations, à nous communiquer une foule de détails ignorés, enfin à transmettre leurs connaissances à leurs compatriotes : il est honorable à la nation française de n'être pas restée en arrière dans cette tentative. Depuis six ans, nous avons des écoles au Sénégal. [...] plusieurs princes de l'intérieur envoient leurs enfans au chef-lieu de la colonie française. D’un autre côté, les Anglais établissent des écoles, non seulement à l'embouchure de la Gambie, mais à plusieurs centaines de milles du Cap de Bonne-Espérance, et les Cafres, comme les Wolofs, acceptent le bienfait de l'éducation (Dard 1825: xvii, nota)

No se puede dejar de lado el importante papel que jugó la religión en el asentamiento de las naciones europeas en aquellos territorios. Al igual que había ocurrido con los misioneros en América durante el siglo XVI, el siglo XIX vio llegar a las regiones colonizadas misiones protestantes de británicos, misiones católicas de belgas y, desde otra perspectiva, misiones educativas francesas. Pero incluso entre los franceses la religión también estaba presente. En la misma obra francesa de donde hemos tomado la anterior cita, se señala en su portada: «Allez, portez aux Africains les arts et les bienfaits: semez au milieu d'eux la parole de paix et la morale évangélique» ${ }^{4}$.

Ese carácter religioso es el origen que movió a realizar obras lingüísticas sobre aquellas lenguas africanas o asiáticas, pues en ocasiones el objetivo no era otro que poder hacer la traducción de la Biblia a esa lengua nativa y adoctrinar así a los habitantes locales ${ }^{5}$. Hasta conseguirlo, algunos misioneros británicos incluso usaron otros textos como base doctrinal: por ejemplo, El progreso del peregrino (The Pilgrim's Progress) de John Bunyan (1628-1688), obra que había sido publicada en 1678 ( $1 .^{\mathrm{a}}$ parte) y 1684 (2. ${ }^{\mathrm{a}}$ parte), pero que «durante la época

4 Cita tomada de la portada del Dictionnaire français-wolof et français-bambara, [Paris], Imprimerie Royale, 1825 por J. Dard. Hemos consultado el ejemplar de la Bodleian Library digitalizado en Google Books.

5 Así lo dirá Bentley (1887: xxii), del que tratamos después: «I feel I must state that the great thought which was ever before me, and which prompted me to carefulness and thoroughness, was, that I was preparing a grammar and dictionary, which would be used by my colleagues in the translation of the Scriptures, and that, therefore, any carelessness or error might be very serious in its consequences». 
colonial se tradujo a más de 80 lenguas de África, donde ayudó a definir el Imperio británico como un solo espacio cultural. Por si fuera poco, se realizaron 24 traducciones en el sur de Asia, 9 en el sudeste asiático y otras 11 en Australasia y el Pacífico» (Lyons 2011: 118-119)6.

Así, siguiendo a veces el ejemplo de lo que españoles y portugueses habían hecho en los siglos anteriores, estos misioneros decimonónicos compusieron gramáticas y diccionarios de las regiones africanas o asiáticas en las que vivieron. Eran lenguas sin escritura, lo que los situaba al mismo nivel que aquellos misioneros de los siglos XVI y XVII en América. Ahora bien, estos trabajos lingüísticos del siglo XIX no son todos del mismo valor. Como señala Moussay (1997: 8) en la bibliografía de las Missions étrangères et langues orientales:

Les travaux linguistiques des missionnaires ne sont pas tous d'égale valeur. Beaucoup de missionnaires, ignorant les règles de la lexicographie moderne, travaillaient avec le souvenir des dictionnaires latin-français ou grec-français de leurs études antérieures, et se contentaient souvent, quand ils composaient des grammaires, de transposer dans les langues asiatiques les catégories morphologiques des langues européennes.

Las lenguas africanas además suponían un reto: su número era amplio y presentaban poca semejanza tipológica entre sí: «Déjà l'on a remarqué qu'à la différence des langues de l'Asie et de l'Europe, et même de l'Amérique, le continent africain possède une multitude de langues qui ont très peu de rapports ensemble.» (Dard 1825:xv).

La cantidad de obras publicadas sobre lenguas africanas o asiáticas en el periodo comprendido entre 1830-1900 fue considerable, ya fueran realizadas por los británicos o por los franceses, y tuvo detrás una serie de instituciones, sociedades religiosas o geográficas que las fomentaron.

Aquí nos detendremos brevemente en una de esas obras para ver cómo fue su realización y si pudo darse trasposición de los modelos tipológicos de lenguas europeas en su descripción: el Manuel de conversation franco-laotien de Jean Estrade (1895), donde se describe la lengua lao de la Indochina francesa.

6 The Pilgrim's Progress cuenta cómo su personaje, Cristiano, lucha contra la tentación y demás peligros para eliminar los pecados y llegar a la ciudad celestial, donde se une con Dios. «El libro funcionó como una suerte de sucedáneo de la Biblia y fue una útil herramienta pedagógica cristina, sobre todo para instar a la conversión. Los misioneros solían encuadernarlo con partes de la Biblia. [...] El progreso del peregrino se ha traducido a 200 idiomas. [...] Las traducciones de la obra de Bunyan a lenguas no europeas fueron el resultado de complejas negociaciones entre misioneros y jóvenes conversos, que a veces se llamaban language boys (algo así como «muchachos del idioma»). El sentido literal del texto no siempre tenía una fácil traducción a los contextos culturales africanos. Por ejemplo, los protestantes kele del Congo omitían las secciones que explicaban el concepto cristiano del pecado original, pues dicha idea era incompatible con sus supuestos culturales. La historia sufrió también otros cambios para hacerla relevante al público no europeo. A las versiones africanas se añadieron personajes negros» (Lyons 2011: 118-119). 
Hemos manejado también otras obras, como el Dictionnaire français-wolof et français-bambara suivi du Dictionnaire wolof-français de Dard (1825), en cuyo prólogo se describe la lengua bantú del Congo; la de Théodore Roland de Bussy, L'idiome d'Alger, ou dictionnaires français-arabe et arabe-français précédés des principes grammaticaux de cette langue (Alger, Brachet et Bastide, 1838), y la del inglés William Holman Bentley (1855-1905), misionero de la Baptist Missionary Society. Su obra es importante: Bentley realizó uno de los más significativos diccionarios de la lengua kongo o kikongo (lengua hablada hoy por personas de la República del Congo, República Democrática de Congo y Angola), pero, más que su Dictionary and Grammar of the Kongo Language, as spoken at San Salvador, the ancient capital of the old Kongo empire, West Africa (London, Baptist Missionary Society, 1887), nos interesa el apéndice que publicó pocos años después (Appendix to the Dictionary and Grammar of the Kongo language, 1895), porque en su prólogo explica la planta de su obra y cómo fue realizada: es una auténtica joya para conocer cómo se realizaron estos manuales de lenguas decimonónicos, normalmente obras de un solo autor, al estilo de los diccionarios y gramáticas de aquellos misioneros, ya mencionados, del siglo XVI.

Allí describe que existía algún trabajo precedente sobre la lengua de la región de la época de los portugueses (1484), pero apenas ninguna otra fuente, así que tomó como modelo el diccionario de otra lengua vecina que había sido publicado unos años antes. Para conocer la lengua primeramente usó algunos hablantes que sabían algo de portugués como lengua intermediaria, pero posteriormente tuvo que recurrir a niños porque con ellos podía pasar más tiempo. Otras voces adquiría palabras mediante la vida diaria con ellos, a través de trabajos cotidianos, como cortando madera para construir casas o viajando.

The children, however, were always at hand, and the words could be caught more easily from them, as they articulated more distinctly. Every word gained was noted, entered in our memo books, and in the evening we each reported to the other all that had been acquiered, and further discussed the new words. Our vocabulary steadily increased as we talked or played with the children, or initiated our scholars into the mysteries of reading and writing. The alphabet was arranged, and the force of letters decided upon. Some words were gained as we worked with the men who helped us in building our houses, timber cutting, quarrying limestone, or in cutting the canoe to transport the stone to the holes in which the Portuguese had burnt their lime long ago. Also when travelling, or doctoring the sick, we collected words, and thus added daily to our lists. When we were able to speak the language a little, and ask questions about it in the language itself, we made further progress, and many mistakes were corrected. (Bentley 1887 Preface: xiii-xiv)

Una vez recogidas en sus cuadernos un número suficiente de palabras, era precisa la reordenación de las voces y la distribución de las acepciones o combinaciones semánticas con que se usaban las voces, separándolas por letras. Con la ayuda de un nativo comprobó durante varios años que el significado señalado era correcto y no había errores. «For more than five years have I been 
watching carefully for new forms, words, and grammatical constructions, but have never had any reason to suspect changeableness.» (Bentley 1887: xvi)

Luego, ya ordenado todo, lo llevó a imprimir a Inglaterra, pero al repasar el manuscrito se dio cuenta de que debía aprender algo de la técnica lexicográfica y, viendo otros diccionarios de inglés, comenzó a establecer entradas o subacepciones según el régimen de los verbos, etc. Tras ello dio la vuelta al diccionario English-Kongo y creó la dirección Kongo-English, pero antes tuvo que eliminar todas las erratas que se hallaron. Finalmente, supervisó la corrección de las pruebas.

Interesante es también la observación que hace: la gramática y las reglas de la lengua kikongo no tienen excepciones, lo que es notable comparándolo con las lenguas europeas, y trata de justificar por qué:

As we carried our investigations deeper and deeper, we were surprised to note how far-reaching and absolute are all the principles and rules. We are able to state and explain them definitely, and finde them working without exception. Indeed, when an irregularity is found, at once the suspicion is raised that the real principle has not been discovered, and our Grammar stands without the usual lists of exceptions. In this respect it figures as an ideal, impossible to our European languages, which have been subject to such interaction and reaction, until Keltic, Saxon, and Latin rules have brought about results and changes, so arbitrary and strange as to be almost inexplicable, and we have to state facts as facts, and leave them so. In the Kongo language, as spoken at San Salvador, we find a language uninfluenced by any other great family. Negro, Semitic, or Hottentot peoples have been too remote, the nearest being the Negro Etik, sopoken north of the Cameroons, nearly 1,000 miles away. (Bentley 1887 Preface: xix)

La realización del Dictionnaire et guide franco-laotiens o Manuel de conversation de Jean Estrade ${ }^{7}$ ([Toulouse, Impr. Berthoumieu], 1895, 2. ${ }^{\text {a }}$ ed.) debió de ser similar a la de Bentley. La obra surgió por la necesidad de tener que comunicarse con los nativos sin intérpretes, así que Estrade reunió las palabras y expresiones de la lengua lao más usuales (lo cual es, recordemos, lo que ya señalaba Núñez de Balbuena en su vocabulario francés árabe: «les mots les plus usuels et les plus nécessaires à connaître, lorsqu'on voyage dans un pays quelconque dont on ignore la langue»). Se trata propiamente de un trabajo lingüístico, pero, por exigencias editoriales que se señalan en el prólogo, pero que no se especifican («certaines circonstances»), la publicación debió incluir aspectos de guía turística: de aquí el mapa de itinerarios por el bajo Laos y y las noticias culturales. La tipografía en lengua lao no existía salvo en la imprenta

7 Estrade, que era médico de la Marina, publicó también un informe titulado Aperçu hygiénique sur le Bas-Laos: Notes médicales, 1893-1894-1895, Pamiers, Impr. de Mme Delaye, 1896, 42 p. Existen ejemplares en las bibliotecas Mazarina y SainteGeneviève de París. En la biblioteca del Ministère des Affaires Étrangères de París hay otro título que se le atribuye: Le Japon: ses voies et moyens de communication intérieurs et extérieurs, Paris, A. Rousseau, 1905. 
Nazareth de Hong Kong, así que los caracteres tuvieron que reproducirse a mano en hojas separadas de las impresas.

El plan de la obra comprende los siguientes apartados: descripción de los signos del alfabeto; cómo leer la pronunciación figurada; nociones sobre el idioma; reglas gramaticales. Sigue una primera parte formada por una nomenclatura con 16 epígrafes que se pueden agrupar en los siguientes puntos:

- Días de la semana. Meses;

- Puntos cardinales;

- Jerarquía laociana;

- Números. Monedas. Pesos;

- Principales frutas de Laos;

- La familia;

- Los animales;

- Diferentes partes del cuerpo humano;

- Términos relativos al tiempo;

- Árboles y plantas;

- Adverbios, preposiciones, conjunciones...;

- Condados, provincias, ciudades, pueblos, ríos;

- Medidas lineales.

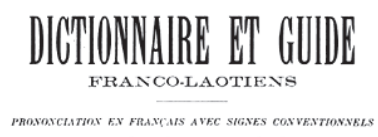

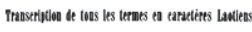

MANUEL DE CONVERSATION

LO DOoteur HETRADH
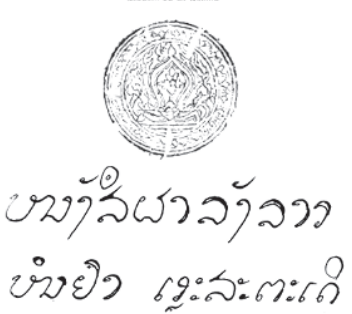

DEUXIÉME EDITION

1888

\section{II. - Mois de l'Année}

1. Décembre

2. Janvier

3. Février

4. Mars

5. Avril

6. Mai

7. Juin

8. Juillet

9. Août

10. Septembre

11. Octobre

12. Novembre dueun tieng. dueun yi. dueun sam? dueun sidueun ha. dueun hoc! dueun tièt. dueun pèt. dueun kaou. dueun sip. dueun sip èt. dueun sip song.

L'année laotienne commence au mois de décembre. La lune (dueun) est le terme principal dans l'évaluation du temps.

Jean Estrade, Manuel de conversation, 1895.

Portada y muestra de la nomenclatura (p. 24)

Una segunda parte recoge el vocabulario alfabético, que es sin duda lo fundamental de la obra, pues abarca las p. 51-270 (unas 3500 palabras) y una tercera las expresiones usuales, los términos y diálogos relativos a viajes en piragua, a viajes por tierra y algunas muestras de las frases más usuales. Por último, una cuarta parte recoge notas sobre ciertas palabras u observaciones etnográficas (como el parto, los aspectos religiosos de sus habitantes, el cultivo de especias, el corte de pelo de las mujeres al casarse, ritos funerarios, etc.). 


\section{EXPRESSIONS USUELLES}

1. Où allez-vous?

2. D'où venez-vous?

3. Je vais manger

4. J'ai mangé

5. Quelle heure est-il?

6. Venez demain

7. Partez vite

8. Comment vous portezvous?

9. Je vais bien

10. Voulez-vous boire?

11. Il n'y en a pas

12. Non certes (très négatif)

13. Je ne comprends pas

14. Fumez une cigarette

15. Donnez-moi une allumet ${ }^{t \text {. }}$

16. Je n'aime pas cela pay say.

ma tè say.

pay kinn khàou.

kinn khâou lèo.

tiac mông.

ma meuhun.

pay vay vay.

than you di bo?

khoy you di.

yak kinn bo.

bo $\mathrm{mi}$.

bo mèn dok.

bo houtiac (légère élision de hou).

doud kok ya - !

hay khoy may khit fay?

ho sob an ni.

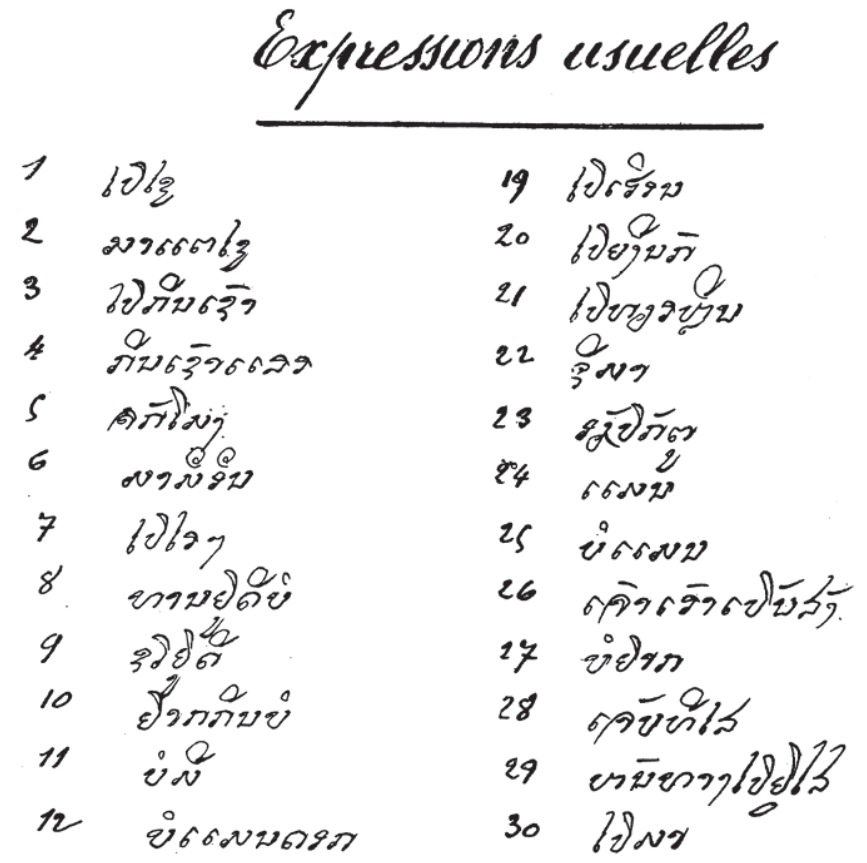

Estrade, Manuel de conversation, 1895. Expresiones habituales y sus equivalencias laos manuscritas (p. 272) 


\section{TERMES ET DIALOGUES}

Sc rapportant aux voyages par terre

1. Y a-t-il une route de Kong à Bokham?

2. Est-ceune routeà charrettes?

3. Ya-t-il des prèks à traverser?

4. Sont-ils grands?

万. Y a-t-il beaucoup d'ean?

6. Combien de jours y a-t-il de Kong à Bokham?

i. Passe-t-on par beaucoup de villages?

8. Je veux dix coolies et deux charrettes à bœufs

9. Donne\%-moi un guide jus(qu'au prochain village

10. Nous partirons demain à la première heure
Koung mi hon thang pay Bôkham bo.

hon thang ni kièn ngoua? pay day bo?

mi houhai pay nam bo?

péll gnay bo?

mi nâm lay bo ?

Koung pay Bôkham tiac mu. hon thang Bôkham mi ban lay bo?

khoy yak day couli sip khon kap kièn ngoua? song lam. hay .nay thang khoy p'hou nung nam khoy pay thung ban na.

si pay meuhun ta deuc.

bermes et dialogues ise rappsortant aux voyages pare terre

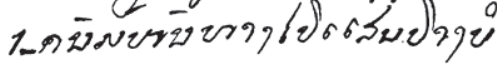

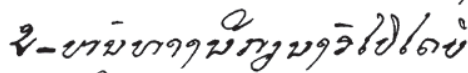

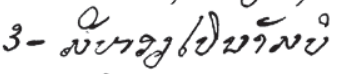

4- 6 2)

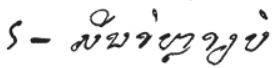

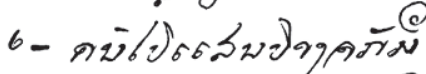

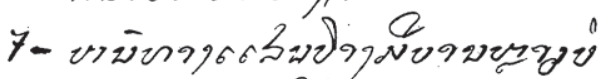

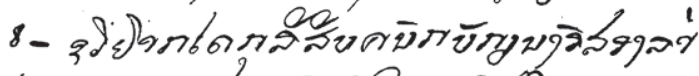

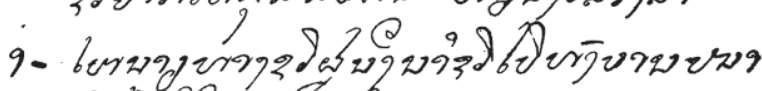

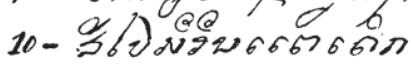

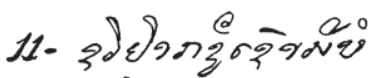

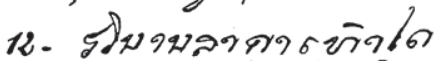

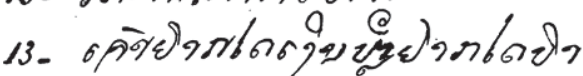

Estrade, Manuel de conversation, 1895. Expresiones habituales y sus equivalencias laos manuscritas (p. 272) 
La descripción de la lengua lao suponía un reto, al ser tan diferente de las tipologías de lenguas europeas: se trata de una lengua tonal, donde la pronunciación hace cambiar el significado de las palabras, y con verbos invariables a los que se añaden partículas para designar futuro o pasado.

Las diferencias culturales son patentes asimismo en la diferente concepción temporal: para los laosianos de finales del XIX, el día empezaba a las 6 de la mañana y terminaba a las 6 de la tarde, de modo que las 9 horas de la tarde serían para ellos las 3 de la mañana. Del mismo modo, la división del día no se regía por las horas, sino por la posición del sol:

Très peu de Laotiens ont la notion du temps divisé en heures. Lorsqu'on leur ordonne de partir à telle heure de la journée il est prudent, si l'on veut être sûr d'être compris, de leur indiquer l'endroit où le soleil se trouvera au moment du départ. (Estrade 1895: 15)

Lo interesante de la obra es que reúne en un solo volumen las varias partes requeridas para el aprendizaje de lenguas: cuestiones de pronunciación, aspectos gramaticales, vocabulario y cuestiones culturales.

\section{Conclusiones}

Hemos realizado un acercamiento a obras similares en lo que se refiere a su cronología y finalidades lingüísticas -necesidades comunicativas-, pero desarrolladas con planteamientos muy diferentes y en contextos geográficos y culturales muy distintos.

Por un lado, las guías de conversación europeas mostraban un mismo contexto cultural, una intercomprensión similar en todos los lugares (a pesar de las diferencias regionales existentes entonces, mucho mayores que ahora). Los viajeros eran burgueses, tomaban el tren, el barco o los caminos y los destinos eran fundamentalmente ciudades. No se suele dar en esas obras nociones gramaticales o fonéticas, pero sí frases dialogadas: se busca la equivalencia, no la explicación, y se emplean para ello oraciones, textos estándares y modelos de cartas con los que comunicarse desde un punto de vista práctico.

Lo visto en las obras coloniales coloniales muestra que tanto franceses como ingleses comenzaron su interés hacia las lenguas de esas regiones colonizadas a través del léxico. A partir de ello construyeron diccionarios y, basándose en las voces, ocasionalmente establecieron o dedujeron los criterios gramaticales de esa lengua (las cuestiones gramaticales no necesariamente están, o bien suelen aparecer en los prólogos). El interés descriptivo era el principal, pero hay que recordar no eran lexicógrafos, sino misioneros, y tenían intereses religiosos o particulares.

Las obras que hemos descrito aquí, tanto la de Bentley sobre el kikongo como la de Estrade sobre el lao, permiten verificar la influencia de los métodos usados en las lenguas europeas en las descripciones sobre las lenguas colonizadas: se incluyen apartados breves de nomenclaturas y léxico frecuente, se recogen las 
expresiones usuales o de primera necesidad. Parece evidente que los diálogos y los fragmentos de frases vigentes en los manuales europeos de época fueron usados posiblemente como el modo más cercano a las necesidades de comunicación, al igual que siguen siendo necesarias en nuestros días cuando viajamos a regiones con lenguas que desconocemos. La diferencia fundamental estriba en que lenguas tipológicamente tan diferentes obligaban al europeo a realizar una breve descripción, pero nunca se llegaba a una profundización tan grande como sería precisa para codificar el sistema gramatical de la lengua (cada obra puede presentar una estructura diferente, lógicamente, y para un mejor estudio sería necesario comparar más títulos de los aquí mostrados).

Otra conclusión que extraemos es que el modo de trabajo para la realización de estas obras de regiones colonizadas fue bastante similar al de la lingüística misionera del siglo xvI: no existían materiales previos, o eran escasos, y la información debía ser obtenida de primera mano frente a los hablantes. En el caso de estas obras del siglo XIX, representan una etapa importante en la historia de las lenguas europeas y de la lingüística general, al poner en contacto nuevas lenguas con estructuras y concepciones mentales diferentes. El interés lingüístico hacia estas lenguas sería un paso hacia la lingüística comparada.

\section{Bibliografía}

Bentley W.H., 1887, Dictionary and Grammar of the Kongo Language, as Spoken at San Salvador, the ancient capital of the old Kongo empire, West Africa, London, Baptist Missionary Society.

Bentley W.H., 1895, Appendix to the Dictionary and Grammar of the Kongo Language, as Spoken at San Salvador, the ancient capital of the old Kongo empire, West Africa. Compiled and prepared for the Baptist Mission on the Kongo River, West Africa by the Rev. W. Holman Bentley, Missionary of the Baptist Missionary Society on the Kongo, London, Published by the Baptist Missionary Society and Kegan Paul, Trench, Trübner \& Co.

Dard J., 1825, Dictionnaire français-wolof et français-bambara suivi $d u$ Dictionnaire wolof-français par M. J. Dard, ancien élève du Musée d'Histoire Naturelle de Paris, ancien instituteur de l'école du Sénégal, ex-professeur de Mathématiques et de Navigation, Maître de pension à Bligny-sous-Beaune, [Paris], Imprimerie Royale.

Estrade J., 1895, Dictionnaire et guide franco laotien: prononciation en français avec signes conventionnels, transcription de tous les termes en caractères laotiens, manuel de conversation par le Docteur Estrade... 2 édition, [Toulouse, Impr. Berthoumieu].

Lyons M., 2011, Libros: Dos mil años de historia ilustrada, Barcelona, Lunwerg.

Moussay G. et al., 1997, Missions étrangères \& langues orientales: contribution de la société des missions étrangères à la connaissance de 60 langues d'Asie: bibliographie de 1680 jusqu'à 1996, Paris, L'Harmattan. 
Núñez de Taboada M.M., 1833, Guide de la conversation, ou, Vade-mecum du voyageur: en sept langues, anglais, allemand, français, italien, espagnol, russe, barbaresque ou moresque; contenant des Conversations familières sur divers besoins et circonstances où il peut se trouver. Ouvrage mis en ordre, revu et corrigé sous la direction de M. Nuñez de Taboada, Directeur de l'établissement d'Interprétation générale des Langues, et auteur d'un Dictionnaire françaisespagnol et espagnol-français qui porte son nom. Suivi d'un Vocabulaire français arabico-barbaresque, et d'un Dialogue dans ces deux langues sur des choses de première nécessité, Paris, Thiériot, Libraire.

Roland de Bussy Th., 1838, L'idiome d'Alger; ou, dictionnaires français-arabe et arabe-français précédés des principes grammaticaux de cette langue, Alger, Brachet et Bastide.

Traveller's Manual of Conversation in Four Languages, English, French, German, Italian. With Vocabulary, short questions, etc., 1875, 22nd ed., Leipzig, K. Baedeker. 